\title{
Can the memory of an object be enhanced by imagining its loss?
}

\author{
LI Qi ${ }^{1}$, QI Yue ${ }^{2,3}$, LIU XianYun ${ }^{4} \&$ LUO Jing ${ }^{5,6^{*}}$ \\ ${ }^{1}$ Key Laboratory of Behavioral Science, Institute of Psychology, Chinese Academy of Sciences, Beijing 100101, China; \\ ${ }^{2}$ State Key Laboratory of Brain and Cognitive Science, Institute of Psychology, Chinese Academy of Sciences, Beijing 100101, China; \\ ${ }^{3}$ Graduate University of the Chinese Academy of Sciences, Beijing 100049, China; \\ ${ }^{4}$ Academy of Psychology and Behaviour, Tianjin Normal University, Tianjin 300387, China; \\ ${ }^{5}$ Department of Psychology, Capital Normal University, Key Laboratory of Study and Cognition of Beijing, Beijing 100048, China; \\ ${ }^{6}$ Key Laboratory of Mental Health, Institute of Psychology, Chinese Academy of Sciences, Beijing 100101, China
}

Received July 9, 2012; accepted October 30, 2012; published online January 21, 2013

\begin{abstract}
Previous investigations examining salient memories have demonstrated that personal memories that are important to individuals and contain emotional information are better remembered than neutral events. Using behavioral and brain-imaging experiments, the present studies explored whether the previous finding was applicable imagined loss. In a behavioral experiment, a free recall paradigm was used to compare the memory performance of individuals who imagined loss with that of individuals who imagined importance. The superior memory performance conferred by imagining loss was constrained to ordinary items of low to medium importance and did not generalize to vital items. Moreover, brain imaging evidence revealed that the activation in certain brain regions was stronger when participants were imagining the loss of ordinary items of low to medium importance compared to vital items. These brain regions included cognitive effort-related areas (such as the parietal cortex and middle prefrontal cortex) and areas related to emotional experiences and emotion-related memories (such as the amygdala, parahippocampal gyrus, and posterior cingulate gyrus). Our study provides a new way of exploring the superior memory performance when imagining loss and enriches the literature on memory enhancement by contributing to a deeper understanding of the psychological mechanisms related to the imagining of vital losses.
\end{abstract}

imagining, vital loss, memory enhancement effect, fMRI

Citation: $\quad$ Li Q, Qi Y, Liu X Y, et al. Can the memory of an object be enhanced by imagining its loss? Chin Sci Bull, 2013, 58: 1767-1774, doi: 10.1007/s11434-012-5659-0

Prior investigations examining salient memories have demonstrated that personal memories are important to individuals and contain emotional information are better remembered than neutral events [1]. Research has also revealed that the more closely an object is related to the self, the better it is remembered. The best memory recall is observed for information about one's self, followed by information about one's mother and friends, whereas the poorest memory performance is observed for information about strangers [2]. This phenomenon occurs because people pay more attention to self-referential information, which promotes the organization and elaboration of memories. For example, women typically have better memory perfor-

*Corresponding author (email: luoj@psych.ac.cn) mances for weight-related words given that they care more about their weight than men. Men often show better memory performance for muscle-related words given that they pay more attention to their muscle strength [3]. Additionally, individuals are more likely to remember emotional information compared with information that is lacking in emotional importance. Abundant evidence from source memory paradigms and remember-know procedures indicates that individuals' memories are more detailed with regard to emotional information than for neutral information [4]. Neuroimaging studies have implicated the prefrontal cortex and medial temporal areas in the successful encoding of material into episodic memory [5]. The amygdala is also activated when emotional memories are recalled [6]. Imaging studies also emphasize that the amygdala's arous- 
al-mediated influences and its interactions with brain structures in the frontal and temporal lobes enhance memories. From an evolutionary perspective, emotional memory plays a vital role in the survival and reproduction of living beings. Therefore, events of an emotional nature are instrumental to the survival of individuals due to their being better remembered [7].

Converging evidence has shown that emotion can be elicited and studied through the use of hypothetical scenarios [8-13]. One question is whether imagining the loss of more important things triggers stronger negative emotions, which then results in stronger memory-enhancing effects. To address this question, we designed two experiments. In the first experiment, participants were assigned to either a loss group or an importance group. In an initial encoding stage, participants in the loss group were asked to imagine and experience how distressed they would feel if they lost an item, whereas participants in the importance group were asked to think of how important that item was in their life. During the memory retrieval stage, the two groups completed free recall tests, and the memory performances of the two groups were analyzed. In the second experiment, we investigated the neural mechanisms underlying the imagined loss of vital objects using functional magnetic resonance imaging (fMRI). Based on their level of importance, the items presented in the experiments were divided into the following three categories: (i) high level $(\mathrm{H})$ for vital objects (e.g., eyes); (ii) medium level (M) for items of medium importance (e.g., glasses); and (iii) low level (L) for items of low importance (e.g., a table lamp).

In the present study, we made three predictions regarding salient memory. First, the memory performances for both the importance group and the loss group should follow the rule that the more important the object, the better the recall. In other words, participants should have the best memory performance for vital objects, a moderate performance for objects of medium importance, and the worst performance for items of low importance. Second, given that emotional information is better remembered than non-emotional information in long-term memory, the memory performance of the loss group should be better than that of the importance group. Third, in the loss group, the activation of the medial prefrontal cortex, which is involved in processes of self-representation [14] and cognitive control [15], and the amygdala, the parahippocampal gyrus, and the posterior cingulate gyrus, which are associated with emotional experience and emotional memory [16-18], should become stronger as the importance of the imagined objects increases.

\section{Methods}

\subsection{Participants}

All of the participants were healthy university students who reported a lack of neurological or psychiatric history. Each participant voluntarily enrolled in the study and signed an informed consent form prior to the experiment. The Institutional Review Board of the Institute of Psychology, the Chinese Academy of Sciences approved the experimental procedure.

Seventy students participated in Experiment 1 (17 males, $21.56 \pm 0.25$ years $)$, and 28 students participated in Experiment 2 ( 9 males, $22.93 \pm 0.48$ years).

\subsection{Stimuli}

We carefully selected 1100 high-frequency Chinese nouns, each of which consisted of two characters [19]. Then, the relative importance, familiarity, and concreteness of each noun were rated by a separate group of 35 participants on a 7-point scale (1 being unimportant, unfamiliar or abstract and 7 being extremely important, familiar or concrete). Importance was defined as how desirable or valuable the item was to each individual's life, and concreteness was defined such that an extremely concrete image uses words that can be experienced as sensations or feelings. The final stimulus set consisted of three categories of nouns.

For Experiment 1, the set consisted of 15 vital nouns with high importance ratings (mean $\pm \mathrm{SE}=6.26 \pm 0.06$ ), 15 trivial nouns with medium ratings (mean $\pm \mathrm{SE}=4.39$ \pm 0.11 ), and 15 trivial nouns with low ratings (mean $\pm \mathrm{SE}$ $=2.95 \pm 0.99$ ). For Experiment 2, the set consisted of 20 vital nouns with high importance ratings (mean $\pm \mathrm{SE}=$ $5.91 \pm 0.08$ ), 20 trivial nouns with medium ratings (mean $\pm \mathrm{SE}=4.02 \pm 0.04$ ), and 20 trivial nouns with low ratings (mean $\pm \mathrm{SE}=2.72 \pm 0.14$ ). When the frequency, number of strokes, familiarity and concreteness ratings were held constant $(P s>0.05)$, there was a significant difference in the importance ratings between any two categories ( $P S$ $<0.001)$.

\subsection{Procedure}

In a double-blind design, the participants in Experiment 1 were assigned to either the loss group or the importance group. The participants were exposed to two specific "loss" examples in the loss group and two specific "importance" examples in the importance group. The first loss example was the "Loss of cola". Participants first drank a small amount of cola and described its flavor. They were then told that they would not get to drink cola ever again if the word "cola" was presented on the screen. The second loss example was the "Loss of eyes". Participants were told that they would be blind for the rest of their lives if the word "eyes" was presented on the screen. We used black cloths to cover participants' eyes and asked them to blindly search for shuttlecocks in a room. During this session, we told the participants that this task was neither a game nor an ability test; rather, it was being conducted to make them imagine and 
experience the feeling of losing their eyes. In the importance group, participants were only instructed to imagine and experience the importance of cola and their eyes in their lives and to report on their experiences.

Experiment 1 consisted of the following three parts: (i) encoding, (ii) memory interference, and (iii) memory retrieval. During the initial encoding stage, 49 Chinese words were presented. To avoid primacy and recency effects due to the presentation order, four words were presented at the beginning and end of the encoding stage, which were not included in the data analysis. Each trial began with a 10-s presentation of a word. Participants were told either to imagine and experience the distress of losing this item (loss group) or to imagine how important this item was in their lives (importance group). A fixation cross was shown during the interstimulus interval for $2 \pm 1 \mathrm{~s}$. The three types of words (H, M, and L conditions) were presented using a Latin Square experimental design. Each word subtended a visual angle of $2.11^{\circ} \times 4.11^{\circ}$. During the memory interference stage, a three-digit number was presented, and participants were asked to continually subtract 3 from this number while saying the calculation out loud for $1 \mathrm{~min}$. In the memory retrieval stage, participants performed a free recall task for 10 min that included 45 words (15 high importance words, 15 medium importance words and 15 low importance words).

At the end of the experiment, participants in the loss group were asked to retrospectively recall and describe their emotional states while they were imagining the loss of the low, medium, and high importance items. The evaluations of the emotional state included the participants' desire to escape imagining the loss and their escape behavior. These two items were evaluated on a 7-point scale ("1": not at all to "7": extremely) with the order of these measures counterbalanced. We provided the list of items from the three conditions to help participants' memory retrieval.

The procedure for Experiment 2 was the same as the one used with the loss group in Experiment 1 except that the memory interference and memory retrieval stages were removed (Figure 1).

\subsection{Image acquisition and analysis}

During MRI scanning, imaging data were obtained with a

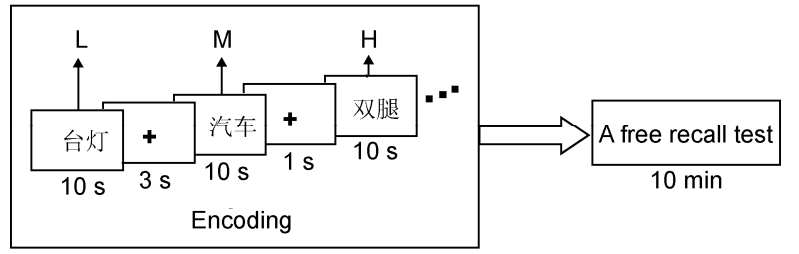

Figure 1 Design for Experiment 1. A depiction of the three different experimental conditions. The Chinese words in the figure, “台灯”, “汽车”, and "双腿”, mean “lamp", “car", and “legs", respectively.
3-T SIEMENS Magnetom Allegra System (Siemens, Erlangen, Germany) using a whole brain $\mathrm{T} 2 *$-weighted gradient echo-planar imaging (EPI) sequence (32 transverse slices covering the entire brain were acquired using a gradient-echo echo-planar pulse sequence, repetition time $=$ $2000 \mathrm{~ms}$, echo time $=30 \mathrm{~ms}$, field of view $=220 \mathrm{~ms}$, flip angle $=90^{\circ}$; matrix $64 \times 64 \times 32$, and spatial resolution, $3.4 \mathrm{~mm} \times 3.4 \mathrm{~mm} \times 3 \mathrm{~mm}$ ) with a standard head coil at the Shanghai Key Laboratory of Magnetic Resonance Imaging to measure changes in blood oxygenation leveldependent (BOLD) signals. Anatomical images were obtained using a standard 3D T1-weighted sequence (matrix $256 \times 256 \times 128$; spatial resolution, $0.938 \mathrm{~mm} \times 0.938 \mathrm{~mm}$ $\times 1.33 \mathrm{~mm}$, repetition time $=2530 \mathrm{~ms}, \mathrm{TI}=1100 \mathrm{~ms}, \mathrm{TE}=$ $3.98 \mathrm{~ms}$ ).

We used the statistical parametric mapping software SPM (Version 5; Welcome Department of Cognitive Neurology, London, UK) for image preprocessing and statistical analyses. Prior to preprocessing, the first five functional EPI volumes were discarded to allow for $\mathrm{T} 1$ stabilization. The remaining functional images were realigned to the first scan to correct for head movement. Subsequently, these images were normalized to a standard EPI template at the Montreal Neurological Institute (MNI) with a $3 \mathrm{~mm} \times 3 \mathrm{~mm} \times 3 \mathrm{~mm}$ resample voxel size. Then, the normalized images were spatially smoothed using a Gaussian filter with an 8-mm full width half maximum (FWHM) to decrease differences in individual structural brain anatomy and to increase the signal-to-noise ratio.

To assess the neural activity associated with the three experimental conditions, the three loss decision conditions were separately modeled using a box-car function and convolved with the canonical hemodynamic response function that is built into SPM5. Images were high-pass filtered (128 s) to remove low-frequency signal drifts. A first-order autoregressive model (AR-1) was used to estimate the temporal autocorrelations by using restricted maximum likelihood estimates of variance components.

In the statistical analysis of the fMRI data, experimental conditions $\mathrm{L}, \mathrm{M}$ and $\mathrm{H}$ were defined as three separate regressors on the first level. We separately contrasted the L, $\mathrm{M}$, and $\mathrm{H}$ conditions with a fixation condition. For the second level of analysis, these contrast parameter estimates were submitted to a full factorial group analysis (ANOVA: a one-way analysis of variance) to allow for population inference using a random-effect model. To find regions that are commonly active during the processing of any imagined loss, we performed a conjunction analysis of the L-H and M-H conditions. During the whole-brain search, all of the results from the random effect analysis were thresholded at a combined voxel and cluster-size threshold of $P<0.001$ (uncorrected) and a cluster extent of $k>20$. Unless otherwise specified, we only reported clusters that were significant at $P<0.05$ (corrected for multiple non-independent comparisons). 


\section{Results}

\subsection{Behavioral results}

Figure 2 presents the recall accuracy of the loss and importance groups for each stimulus type. A mixed model ANOVA was applied to these data, and the $2 \times 3$ factors were group type (importance group, loss group) and stimulus type (low, medium, high). The main result was a significant effect of stimulus type $(F(2,136)=57.96, P<0.001)$ and group type $(F(1,68)=6.33, P<0.05)$. There was also a significant interaction between group type and stimulus type $(F(2,136)=6.86, P<0.01)$. Further tests revealed significant differences $(P s<0.001)$ in the accuracy levels of the importance group: $\mathrm{L}($ mean $\pm \mathrm{SE}=36.19 \pm 2.54), \mathrm{M}$ (mean $\pm \mathrm{SE}=47.81 \pm 1.90)$ and $\mathrm{H}($ mean $\pm \mathrm{SE}=64.38 \pm 2.58)(P s$ $<0.001)$. In the loss group, the accuracy of the $\mathrm{L}$ condition (mean $\pm \mathrm{SE}=47.24 \pm 2.24$ ) was significantly lower than the $\mathrm{M}($ mean $\pm \mathrm{SE}=55.81 \pm 1.82)$ and $\mathrm{H}($ mean $\pm \mathrm{SE}=61.52 \pm$ $2.08)$ conditions $(P s<0.01)$; however, there was no significant difference between the $\mathrm{M}$ and $\mathrm{H}$ conditions $(P=0.08)$. Furthermore, in the $\mathrm{L}$ and $\mathrm{M}$ conditions, the accuracy in the loss group was significantly higher than in the importance group $(P s<0.01)$, yet there was no significant difference between the two groups $(P=0.39)$ in the high importance condition.

The self-reported escape desire and behavior ratings for the imagined loss are shown in Figure 3 for the three conditions in Experiment 1. A separate repeated measures one-way analysis of variance (ANOVA) was conducted to analyze the average escape desire and behavior ratings for the three ( $\mathrm{L}, \mathrm{M}$, and $\mathrm{H}$ ) imagined loss conditions.

The imagined loss condition had a significant effect on the escape desire ratings $(F(2,68)=498.91)$ and the escape behavior ratings $(F(2,68)=120.19)(P<0.001)$. Furthermore, the differences in the escape desire ratings for the imagined loss were significant $(P<0.001)$ between conditions, as follows: the L condition (mean $\pm \mathrm{SE}=2.34 \pm 0.15$ ), the $\mathrm{M}$ condition (mean $\pm \mathrm{SE}=3.68 \pm 0.12$ ) and the $\mathrm{H}$ condition (mean $\pm \mathrm{SE}=6.21 \pm 0.11, P s<0.001$ ). The differences in the escape behavior ratings were also significant $(P<0.001)$ between the condition values, as follows: the $\mathrm{L}$

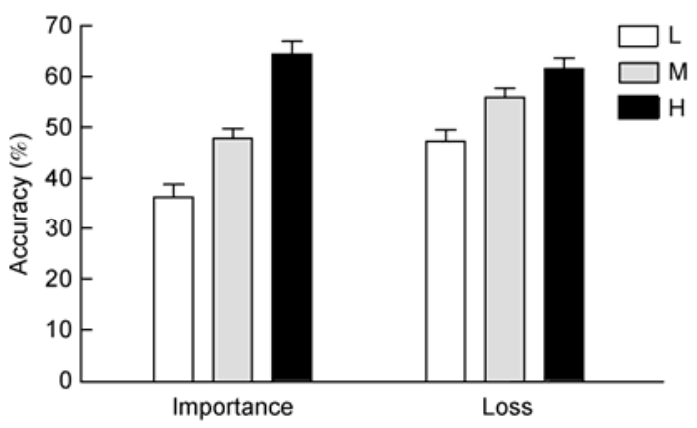

Figure 2 Accuracy (percentage correct) for the loss and importance groups for each stimulus category in Experiment 1.

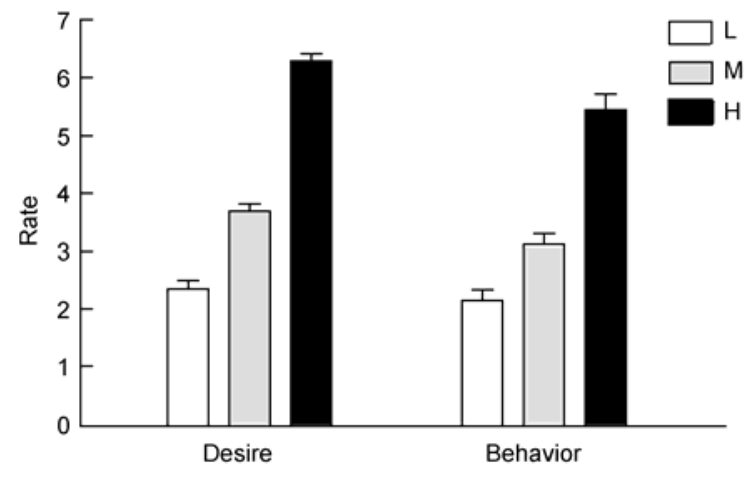

Figure 3 Self-reported ratings of escape desire and escape behavior in each loss imagination condition in Experiment 1.

condition (mean $\pm \mathrm{SE}=2.13 \pm 0.17$ ), the $\mathrm{M}$ condition (mean $\pm \mathrm{SE}=3.24 \pm 0.18$ ) and the $\mathrm{H}$ condition (mean $\pm \mathrm{SE}$ $=5.45 \pm 0.28, P s<0.001)$. Further analysis found that the self-reported ratings values for escape desire and escape behavior were significantly positively correlated $\left(r_{\mathrm{H}-\mathrm{M}}=\right.$ $\left.0.584, r_{\mathrm{H}-\mathrm{L}}=0.577, P s<0.001\right)$. Here, H-M represents the difference between high and medium importance values, and $\mathrm{H}-\mathrm{L}$ represents the difference between high and low importance values. Moreover, the self-reported ratings values for escape behavior and participants' performance on the free recall test were significantly negatively correlated $\left(r_{\mathrm{H}-\mathrm{M}}=-0.362, P<0.05\right)$.

The self-reported escape desire and behavior ratings for imagining loss in Experiment 2 are shown in Figure 4. Separate repeated measures one-way analyses of variance (ANOVA) were conducted to analyze the average escape desire and behavior ratings in the three imagined loss conditions.

The imagined loss condition had a significant effect on the escape desire ratings $(F(2,54)=31.38)$ and the escape behavior ratings $(F(2,54)=9.21)(P<0.001)$. For the escape desire ratings, the $\mathrm{H}$ condition (mean $\pm \mathrm{SE}=5.39 \pm$ $0.29)$ was significantly $(P s<0.001)$ greater than both the $\mathrm{L}$ condition (mean $\pm \mathrm{SE}=3.36 \pm 0.23$ ) and the $\mathrm{M}$ condition (mean $\pm \mathrm{SE}=3.79 \pm 0.28$ ); however, no significant difference was observed between the $\mathrm{L}$ and $\mathrm{M}$ conditions. For the

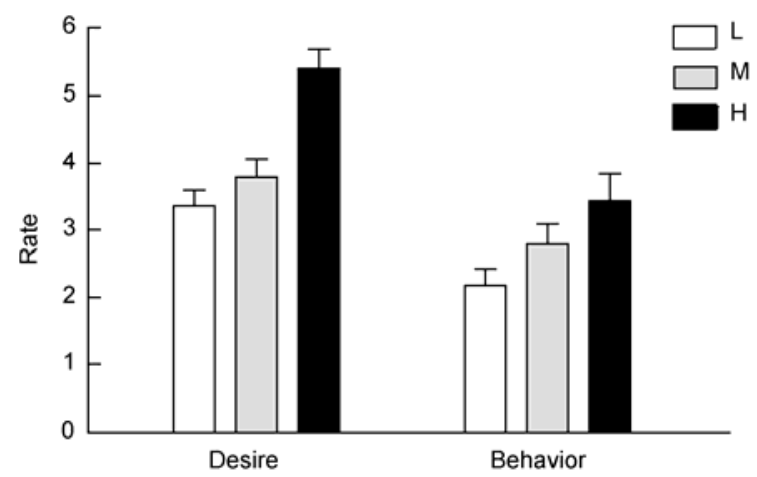

Figure 4 Self-reported ratings of escape desire and escape behavior in each loss imagination condition in Experiment 2. 
escape behavior ratings, the $\mathrm{H}$ condition (mean $\pm \mathrm{SE}=3.43$ $\pm 0.40)$ was significantly $(P s<0.001)$ greater than the $\mathrm{L}$ condition (mean $\pm \mathrm{SE}=2.18 \pm 0.24$ ), yet no significant difference was observed between the $\mathrm{M}$ condition (mean $\pm \mathrm{SE}$ $=2.79 \pm 0.30)$ and either of the other conditions.

\subsection{Imaging results for Experiment 2}

Neural activation associated with vital loss-related imagining. To identify the brain regions that are specifically associated with imagining vital loss, we contrasted the $\mathrm{H}$ condition with the $\mathrm{L}$ and $\mathrm{M}$ conditions. There were no significant clusters when comparing the $\mathrm{H}$ and $\mathrm{L}$ conditions or the $\mathrm{H}$ and $\mathrm{M}$ conditions. In contrast, comparisons revealed significant clusters distributed across a wide network (including the parahippocampal gyrus, inferior parietal lobe, posterior cingulate gyrus (PCC), medial temporal lobe (MTL), rostral anterior cingulate cortex (rACC) and medial prefrontal cortex) when comparing the $\mathrm{L}$ and $\mathrm{H}$ conditions (Figure 5(a), Table 1). Furthermore, a contrast of the $\mathrm{M}$ and $\mathrm{L}$ conditions
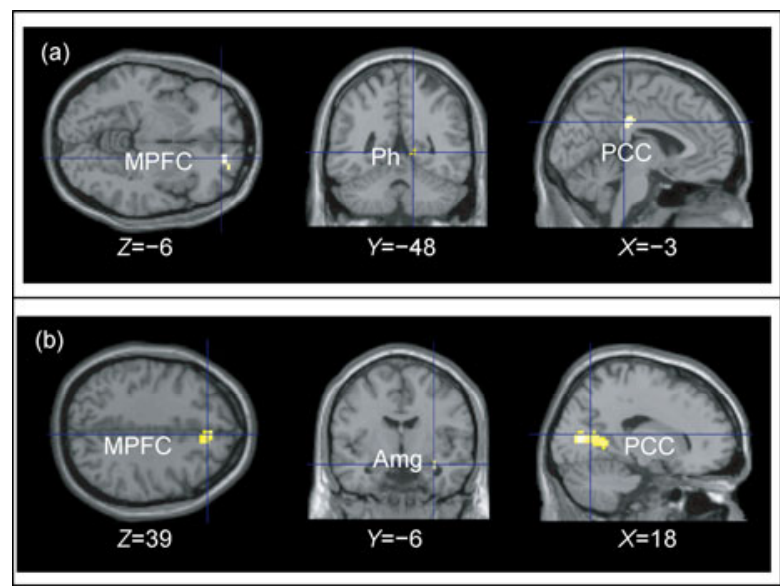

Figure 5 Responses related to imagining vital loss. (a) Selected brain regions show significant activations in the contrast of the $\mathrm{L}$ minus $\mathrm{H}$ conditions, including the medial prefrontal cortex, parahippocampal gyrus, and PCC. (b) Selected brain regions showing significant activations when comparing the $\mathrm{M}$ and the $\mathrm{H}$ conditions, including the medial prefrontal cortex, amygdala, and PCC. MPFC, medial prefrontal cortex; Ph, parahippocampal gyrus; Amg, amygdala; PCC, posterior cingulate gyrus.

Table 1 Brain activations in the $\mathrm{L}$ vs. $\mathrm{H}$ conditions ${ }^{\text {a) }}$

\begin{tabular}{lcrrrrrc}
\hline \multirow{2}{*}{ Brain region } & BA & \multicolumn{7}{c}{ MNI coordinates } & Z-value & Cluster, \\
\cline { 2 - 5 } & & $x$ & $y$ & $z$ & & $K_{\mathrm{E}}$ \\
\hline R. medial PFC & 10 & 18 & 51 & -6 & 3.72 & 21 \\
R. rACC & 32 & 12 & 51 & -15 & 3.31 & \\
R. MTL & 20 & 57 & -45 & -15 & 3.77 & 39 \\
& 20 & 63 & -36 & -18 & 3.52 & \\
R. parahippocampal gyrus & 30 & 15 & -48 & 0 & 3.46 & 124 \\
L. PCC & 31 & -3 & -30 & 33 & 4.15 & 70 \\
R. inferior parietal lobule & 40 & 48 & -42 & 63 & 4 & 194 \\
\hline
\end{tabular}

a) Only clusters (with local maxima coordinates) up to the threshold of $P<0.05$ corrected with 20 or more contiguous voxels were reported. revealed significant activations in the parahippocampal gyrus, amygdala, PCC, MTL, medial prefrontal cortex, and rACC (Figure 5(b), Table 2). Activated regions from the conjunction analysis of the $\mathrm{L}-\mathrm{H}$ and $\mathrm{M}-\mathrm{H}$ conditions are shown in Table 3.

\section{Discussion}

\subsection{Memory was enhanced when imagining the loss of low and medium importance objects}

In the first experiment, the memory performances of the importance group improved as the importance level of a category increased. In the loss group, items of medium importance were recalled more accurately compared with items of low importance. The more important and self-related the information, the better the recall was for the contributions of elaborative and organizational processes [3,20]. The loss group more accurately recalled items of low and medium importance than the importance group. In both conditions, imagining the loss of items triggered negative feelings in the participants, which is consistent with the enhancement of emotional memory; that is, emotional information is better remembered than neural information $[4,21,22]$.

Table 2 Brain activations in the $\mathrm{M}$ vs. $\mathrm{H}$ conditions ${ }^{\text {a) }}$

\begin{tabular}{|c|c|c|c|c|c|c|}
\hline \multirow{2}{*}{ Brain region } & \multirow{2}{*}{$\mathrm{BA}$} & \multicolumn{3}{|c|}{ MNI coordinates } & \multirow{2}{*}{$Z$-value } & \multirow{2}{*}{$\begin{array}{c}\text { Cluster, } \\
K_{\mathrm{E}}\end{array}$} \\
\hline & & $x$ & $y$ & $z$ & & \\
\hline R. medial PFC & 8 & 6 & 36 & 39 & 3.84 & 136 \\
\hline R. dACC & 32 & 12 & 33 & 42 & 3.78 & \\
\hline R. middle PFC & 10 & 39 & 42 & 0 & 4.34 & 77 \\
\hline L. inferior PFC & 46 & -54 & 33 & 12 & 4.24 & 46 \\
\hline \multirow[t]{2}{*}{ L. MTL } & 37 & -63 & -54 & -6 & 3.62 & 161 \\
\hline & 37 & -42 & -60 & 0 & 3.59 & \\
\hline R. parahippocampal gyrus & 36 & 42 & -27 & -21 & 4.17 & 207 \\
\hline R. amygdala & - & 33 & -6 & -18 & 3.74 & 20 \\
\hline R. PCC & 30 & 18 & -63 & 9 & 3.75 & 253 \\
\hline L. PCC & 30 & -18 & -66 & 12 & 4.14 & 64 \\
\hline
\end{tabular}

a) Only clusters (with local maxima coordinates) up to the threshold of $P<0.05$ corrected with 20 or more contiguous voxels were reported.

Table 3 Activated regions from the conjunction analysis of the L-H and M-H conditions ${ }^{\text {a) }}$

\begin{tabular}{lrrrrrl}
\hline \multirow{2}{*}{ Brain region } & BA & \multicolumn{3}{c}{ MNI coordinates } & Z-value & $\begin{array}{c}\text { Cluster, } \\
n_{\mathrm{E}}\end{array}$ \\
\cline { 3 - 6 } R. Cuneus & 23 & 15 & -78 & 12 & 4.5 & 102 \\
R. Lingual Gyrus & 19 & 15 & -54 & 3 & 3.32 & \\
R. MTL & 20 & 57 & -42 & -15 & 3.75 & 18 \\
R. Postcentral Gyrus & 5 & 45 & -42 & 63 & 3.58 & 20 \\
R. middle PFC & 10 & 39 & 45 & 0 & 3.51 & 15 \\
R. medial PFC & 10 & 18 & 54 & -6 & 3.5 & 10 \\
\hline
\end{tabular}

a) Only clusters (with local maxima coordinates) up to the threshold of $P<0.001$ uncorrected with 10 or more contiguous voxels were reported. 
Why were items of medium importance and low importance better recalled by the loss group than by the importance group? Emotional information, which is aroused by imagining loss, is better remembered than a neutral event $[4,21,22]$. For example, individuals' memories were more vivid and detailed for negative words than for neutral words [4]. Negative pictures (compared with neutral pictures) significantly enhanced memory performance. The left amygdala and the right hippocampus were more active during the intentional encoding of negative pictures compared to the encoding of neutral pictures [23]. To our knowledge, this study provides the first demonstration that the improved memory performance that is gained from imagined loss is limited to ordinary objects of low and medium importance. This result supports and enriches the field of salient memory content.

\subsection{Memory performance might not be enhanced by the imagined loss of vital objects}

With regard to salient memory, vital items elicited improved memory performance in the loss group compared with the importance group, which is similar to the result for items of low and medium importance. However, the memory performances of the loss and importance groups were not different for high importance items, and the memory performance of the loss group was not different for high importance and medium importance items. This finding contradicts existing research that suggests that emotional or important personal memories are typically better remembered than general memories [1]. Our behavioral results suggest that recall is not improved by imagining the loss of vital items.

The brain imaging results showed stronger brain activation when people imagined the loss of items of low and medium importance compared with those of high importance. Participants reported having a stronger desire and also described stronger avoidance behaviors to avoid imagining the loss of a vital object than the loss of low or medium importance objects. There were stronger activations in the parietal lobe, medial prefrontal cortex, parahippocampal gyrus, rostral anterior cingulate cortex, and posterior cingulate gyrus when people imagined losing low importance objects compared to high importance objects. Similarly, there were greater activations in the dorsal cingulate cortex, dorsolateral prefrontal cortex, amygdala, parahippocampal gyrus, and posterior cingulate gyrus for the imagined loss of medium importance objects compared to vital objects. The dorsolateral frontal cortex and parietal lobe are important for cognitive control and task difficulty; their activities reflect the cognitive effort of imagined loss processing $[24,25]$. More effort is required to imagine the loss of items of low and medium importance than those of vital importance. The medial prefrontal cortex's activity reflects the processing of self-reference information [14]. This activity indicates that people's imagination might be more closely linked with items in their own lives when they imagine the loss of items of low and medium importance compared with imagining the loss of vital items. The activated areas of the amygdala, rostral anterior cingulate cortex, posterior cingulate gyrus and parahippocampal gyrus process emotional experiences and emotion-related memories $[17,18]$. In particular, the amygdala is well-known for responding to highly threatening and negative information [16], and the rACC is associated with negative utility and emotional conflict [26-28]. Compared with imagining a vital loss, emotional memories and emotion-related experiences were more extensively processed when participants imagined the loss of items of low and medium importance. Conjunction analyses have indicated that the MTL, Middle PFC, and medial PFC were activated when participants were imagining a loss. Functional neuroimaging studies have highlighted the engagement of a common neural network consisting of the medial prefrontal cortex (Brodmann's area BA 10) and MTL (medial temporal lobe) when participants are engaged in imagining future events and remembering past events [29-33]. Therefore, the activations of the MTL, Middle PFC, and medial PFC may be associated with the amount of hard effort needed to imagine a loss during the encoding stage. Based on our brain imaging evidence, memory performance could not be enhanced by imagining the loss of vital items, as was expected.

Why do our study's behavioral and brain-imaging results contradict previous research examining the salient memory of vital objects? Previous studies have found that human beings make scenic costs to maintain their own lives, as well as other vital things, such as wellness, love, glory, justice and personal rights. Each of these things can be regarded as extremely crucial, absolute, scared, and non- negotiable [34-36]. These items may be considered irreplaceable [37,38], and their loss may present a greater threat to the survival and reproduction of human beings than the loss of routine items, such as fortune [39]. The loss of vital items may cause strong feelings of threat or anxiety. People will often use psychological defense mechanisms to avoid or mediate these feelings [40]. These mechanisms may automatically stop or prevent people from imagining emotions that are related to losing vital items, which is consistent with previous studies examining memories of extremely painful incidents. Freud discovered that his patients encountered great difficulty when recalling painful experiences that they had suffered during their childhoods. There seemed to be an enormous power that prevented the memories of such painful experiences from entering people's consciousness; this phenomenon is called "depression" [41]. Anxious people prefer to avoid elaborate processing of negative thoughts during early periods of attention processing. Additionally, anxious individuals remember less about negative items than individuals in control groups [42-45]. Vital things, such as success, self-esteem, and love, are so im- 
portant to people that they often repress memories about their past failures (e.g., losing face, complaints against their lovers, or the names of people who they do not like). Often, people cannot even recall these unpleasant things [46]. Human beings tend to be optimistic and prefer imagining positive things while avoiding negative images. This optimism may trigger adaptive behaviors and improve physical and mental health $[9,47,48]$. Repressing painful or dangerous ideas may be an effective self-protection mechanism $[49,50]$.

The self-reported escape behavior ratings of the loss group increased with the importance level of the items. Participants evoked their psychological defense mechanisms in all three loss conditions, and the degree of defense increased with the importance of the items. Additionally, we analyzed the correlation between the self-reported ratings values for escape with participants' recall performance. The results show that the self-reported ratings values for escape desire and escape behavior were significantly positively correlated. This result indicates that when participants imagined the loss of an important item, the more important that item was, the greater the participants felt the desire to escape, which increases the actual possibility of escape behavior occurring. Moreover, the self-reported ratings values for escape behavior and participants' performance on the free recall test were significantly negatively correlated, which means that the accuracy of recall performance decreases with the increased possibility of escape behavior occurring. A reasonable explanation for this finding is that low and medium importance items are ordinary and easily replaced [39], whereasvital items are irreplaceable [37,38]. Therefore, the psychological defense triggered by the imagined loss of vital items may involve a qualitative change from the defense triggered by items of low and medium importance. This change may suppress an individual's imagination and the emotions related to losing the item. As imagination and emotional arousal decrease, the brain's activation reduces. Thus, in our study, participants' memory performance was not consistent with other salient memory research.

\section{Limitations and prospects}

An important alternative explanation for the inconsistency between our results regarding imagining the loss of vital things and previous salient memory results is that present results may have been caused by a ceiling effect. The term "ceiling effect" refers to the level above which the variance of an independent variable is no longer measured or estimated [51,52]. If the ceiling effect played an important role in memory performance in our study, accuracy of recalled items would have been very high. However, the highest accuracy was only $61.52 \%$, indicating that a ceiling effect did not the cause of the inconsistency between our findings and previous results.
In the present study, an accidental free recall memory test was used to avoid participants to memorize the words for a good performance, rather than to image the loss situation according to the requirement of the task. However, to prevent the floor effect for memeory performance in the different conditions, the number of items to encode was small, making it hard to analyze differences between accurately and inaccurately recalled words by comparing the relevant brain activation areas during the encoding stage in the L, M, and $\mathrm{H}$ conditions. Future research should explore encoding differences in brain areas when either successfully or abortively retrieval items, and among imaging different important items lost at the same time by increasing the number of items. These next steps will help further clarify the mechanism for psychological defense and forgetting.

In summary, our study reveals that recall is enhanced by the imagined loss of low and medium importance items but that this effect does not generalize to vital items. These findings will enrich research on enhanced memory and contribute to a better understanding of the psychological mechanism for the imagined loss of vital items. Furthermore, our findings have great significance for the effective regulation of emotions. Given that imagining loss arouses negative emotions (which always hurt fitness costs) [53], self-defense mechanisms help buffer the effect of these emotions $[40,41]$. Our study has improved the understanding of adaptive behavior and has elucidated emotion regulation.

This work was supported by the National Natural Science Foundation of China (31200782 and 31271079), the Young Scientists Fund of the Institute of Psychology (Y1CX242005), National Key Technologies R\&D Program of China (2012BAI36B01), the Talented Faculty Start-up Funding of Tianjin Normal University (5RW092), the National Basic Research Program of China (2010CB833904), and the Knowledge Innovation Program of the Chinese Academy of Sciences (KSCX2-EW-J-8).

1 Wagenaar W A. My memory: A study of autobiographical memory over six years. Cognit Psychol, 1986, 18: 225-252

2 Symons C S, Johnson B T. The self-reference effect in memory: A meta-analysis. Psychol Bull, 1997, 121: 371-394

3 Unterhalter G, Farrell S, Mohr C. Selective memory biases for words reflecting sex-specific body image concerns. Eat Behav, 2007, 8: 382-389

4 Kensinger E A, Corkin S. Memory enhancement for emotional words: Are emotional words more vividly remembered than neutral words? Mem Cognit, 2003, 31: 1169-1180

5 Otten L J, Henson R N A, Rugg M D. Depth of processing effects on neural correlates of memory encoding. Brain, 2001, 124: 399-412

6 LaBar K S, Cabeza R. Cognitive neuroscience of emotional memory. Nat Rev Neurosci, 2006, 7: 54-64

7 Pitkänen A, Pikkarainen M, Nurminen N, et al. Reciprocal connections between the amygdala and the hippocampal formation, perirhinal cortex, and postrhinal cortex in rat: A review. Ann N Y Acad Sci, 2000, 911: 369-391

8 Hanselmann M, Tanner C. Taboos and conflicts in decision making: Sacred values, decision difficulty, and emotions. Judgm Decis Mak, 2008, 3: 51-63

9 Sharot T, Riccardi A M, Raio C M, et al. Neural mechanisms mediating optimism bias. Nature, 2007, 450: 102-105 
$10 \mathrm{Gu}$ X, Han S. Neural substrates underlying evaluation of pain in actions depicted in words. Behav Brain Res, 2007, 181: 218-223

11 Singer T, Seymour B, O'Doherty J, et al. Empathy for pain involves the affective but not sensory components of pain. Science, 2004, 303: $1157-1162$

12 Osaka N, Osaka M, Morishita M, et al. A word expressing affective pain activates the anterior cingulate cortex in the human brain: An fMRI study. Behav Brain Res, 2004, 153: 123-127

13 Derbyshire S W G, Whalley M G, Stenger V A, et al. Cerebral activation during hypnotically induced and imagined pain. NeuroImage, 2004, 23: 392-401

14 Zhu Y, Zhang L, Fan J, et al. Neural basis of cultural influence on self-representation. NeuroImage, 2007, 34: 1310-1316

15 Miller E K, Cohen J D. An integrative theory of prefrontal cortex function. Annu Rev Neurosci, 2001, 24: 167-202

16 Guyer A E, Lau J Y F, McClure-Tone E B, et al. Amygdala and ventrolateral prefrontal cortex function during anticipated peer evaluation in pediatric social anxiety. Arch Gen Psychiatry, 2008, 65: 1303-1312

17 Touryan S R, Johnson M K, Mitchell K J, et al. The influence of self-regulatory focus on encoding of, and memory for, emotional words. Soc Neurosci, 2007, 2: 14-27

18 Smith A P R, Stephan K E, Rugg M D, et al. Task and content modulate amygdala-hippocampal connectivity in emotional retrieval. Neuron, 2006, 49: 631-638

19 Yuan L. Modern Frequency Dictionary of Chinese Character. Beijing: Yuhang Press, 1990

20 Klein S B, Loftus J. The nature of self-referent encoding: The contributions of elaborative and organizational processes. J Pers Soc Psychol, 1988, 55: 5-11

21 Inaba $\mathbf{M}$, Nomura $\mathbf{M}$, Ohira $\mathrm{H}$. Neural evidence of effects of emotional valence on word recognition. Int J Psychophysiol, 2005, 7: $165-173$

22 Ohira H, Winton W M, Oyama M. Effects of stimulus valence on recognition memory and endogenous eyeblinks: Further evidence for positive-negative asymmetry. Pers Soc Psychol Bull, 1998, 24: 986-993

23 Shigemune Y, Abe N, Suzuki M, et al. Effects of emotion and reward motivation on neural correlates of episodic memory encoding: A PET study. Neurosci Res, 2010, 67: 72-79

24 Altamura M, Elvevåg B, Blasi G, et al. Dissociating the effects of sternberg working memory demands in prefrontal cortex. Psychiat Res-Neuroim, 2007, 154: 103-114

25 Linden D E J, Bittner R A, Muckli L, et al. Cortical capacity constraints for visual working memory: Dissociation of fMRI load effects in a fronto-parietal network. NeuroImage, 2003, 20: 15181530

26 Egner T, Etkin A, Gale S, et al. Dissociable neural systems resolve conflict from emotional versus nonemotional distracters. Cereb Cortex, 2008, 18: 1475-1484

27 Mohanty A, Engels A S, Herrington J D, et al. Differential engagement of anterior cingulate cortex subdivisions for cognitive and emotional function. Psychophysiology, 2007, 44: 343-351

28 Etkin A, Egner T, Peraza D M, et al. Resolving emotional conflict: A role for the rostral anterior cingulate cortex in modulating activity in the amygdala. Neuron, 2006, 51: 871-882

29 Addis D R, Wong A T, Schacter D L. Remembering the past and imagining the future: Common and distinct neural substrates during event construction and elaboration. Neuropsychologia, 2007, 45: 1363-1377
30 Botzung A, Denkova E, Manning L. Experiencing past and future personal events: Functional neuroimaging evidence on the neural bases of mental time travel. Brain Cogn, 2008, 66: 202-212

31 Hassabis D, Maguire E A. Deconstructing episodic memory with construction. Trends Cogn Sci, 2007, 11: 299-306

32 Okuda J, Fujii T, Ohtake H, et al. Thinking of the future and past: The roles of the frontal pole and the medial temporal lobes. NeuroImage, 2003, 19: 1369-1380

33 Szpunar K K, Watson J M, McDermott K B. Neural substrates of envisioning the future. Proc Natl Acad Sci USA, 2007, 104: 642

34 Baron J, Spranca M. Protected values. Organ Behav Hum Decis Process, 1997, 70: 1-16

35 Tetlock P E, Kristel O V, Elson S B, et al. The psychology of the unthinkable: Taboo trade-offs, forbidden base rates, and heretical counterfactuals. J Pers Soc Psychol, 2000, 78: 853-870

36 Tanner C, Medin D L. Protected values: No omission bias and no framing effects. Psychon Bull Rev, 2004, 11: 185-191

37 Grouzet F M E, Kasser T, Ahuvia A, et al. The structure of goal contents across 15 cultures. J Pers Soc Psychol, 2005, 89: 800-816

38 Kasser T. Pain and insecurity, love and money. Psychol Inq, 2008, 19: $174-178$

39 Li Q, Qin S, Rao L L, et al. Can sophie's choice be adequately captured by cold computation of minimizing losses? An FMRI study of vital loss decisions. PLoS One, 2011, 6: e17544

40 Coon D, Mitterer J O. Introduction to Psychology: Gateways to Mind and Behavior. Belmont, CA: Wadsworth Pub Co Press, 2008

41 Freud S. The defence neuro-psychoses. Collected Papers, 1894, 1: 59-75

42 Inaba M, Ohira $\mathrm{H}$. Reduced recollective memory about negative items in high trait anxiety individuals: An ERP study. Int $J$ Psychophysiol, 2009, 74: 106-113

43 Curran T. Effects of attention and confidence on the hypothesized ERP correlates of recollection and familiarity. Neuropsychologia, 2004, 42: 1088-1106

44 Yonelinas A P. The nature of recollection and familiarity: A review of 30 years of research. J Mem Lang, 2002, 46: 441-517

45 Williams J, Watts F, MacLeod C, et al. Cognitive Psychology and Emotional Disorders. Chichester and New York: Wiley Press, 1997

46 Mendolia M. An index of self-regulation of emotion and the study of repression in social contexts that threaten or do not threaten self-concept. Emotion, 2002, 2: 215-232

47 Taylor S E, Brown J D. Illusion and well-being: A social psychological perspective on mental health. Psychol Bull, 1988, 103: 193-210

48 Scheier M E, Carver C S. Dispositional optimism and physical well-being: The influence of generalized outcome expectancies on health. J Pers, 1987, 55: 169-210

49 Horowitz M J, Marmar C, Wallerstein R S. Personality, Styles and Brief Psychotherapy. New York: Basic Books Press, 1984

50 Freud A. The Ego and The Mechanisms of Defence. New York: International Press, 1936

51 Baker H. Illustrated Dictionary of Medical. New Delhi: Lotus Press, 2004

52 Cramer D, Howitt D. The Sage Dictionary of Statistics: A Practical Resource for Students in The Social Sciences. 3rd ed. London: Sage Publications Ltd, 2005

53 Haselton M G, Ketelaar T. Irrational emotions or emotional wisdom? The evolutionary psychology of emotions and behavior. In: Forgas, Joseph P, eds. Affect in Social Thinking and Behavior. New York: Psychology Press, 2006. 21-39

Open Access This article is distributed under the terms of the Creative Commons Attribution License which permits any use, distribution, and reproduction in any medium, provided the original author(s) and source are credited. 